\title{
Advantages of the single nucleotide polymorphism- based noninvasive prenatal test
}

\author{
Kunwoo Kim* \\ Hamchoon Women's Clinic, Seoul, Korea
}

\begin{abstract}
Down syndrome screening with cell-free DNA (cfDNA) in the maternal plasma has recently received much attention in the prenatal diagnostic field. Indeed, a large amount of evidence has already accumulated to show that screening tests with cfDNA are more sensitive and specific than conventional maternal serum and/or ultrasound screening. Globally, more than $1,000,000$ of these noninvasive prenatal tests (NIPTs) have been performed to date. There are several different methods for NIPTs that are currently commercially available, including shotgun massively parallel sequencing, targeted massively parallel sequencing, and single nucleotide polymorphism (SNP)-based methods. All of these methods have their own advantages and disadvantages. In this review, I will focus specifically on the SNP-based NIPT.
\end{abstract}

Key words: Prenatal diagnosis, Single nucleotide polymorphism, High-throughput nucleotide sequencing, Noninvasive prenatal test, Cell free DNA.

\section{Introduction}

The past 20 years has seen remarkable development in the field of prenatal diagnosis, especially with respect to screening for trisomy 21 (Down syndrome). Despite advances in screening tests, the low positive predictive value (PPV <5\%) of the conventional screening test (i.e., use of maternal serum analytes and ultrasound markers, including nuchal translucency) is problematic $[1,2]$. The low PPV is associated with the slew of unnecessary invasive tests performed, including amniocentesis and chorionic villi sampling, which carry a small but significant risk of fetal loss. In 1997, Lo et al. [3] reported the presence of a DNA fragment from the Y-chromosome in maternal plasma, which was found to be derived from pregnant women carrying a male fetus. This fetal cell-free DNA (cfDNA) is thought to originate from the placental trophoblast and then cross from the placenta into the maternal circulation as the cells break down (apoptosis) and the DNA becomes fragmented $[4,5]$. Circulating fetal cfDNA comprises approximately 3-13\% of the total maternal cfDNA. These DNA fragments can be detected as early as 4 weeks of gestation and are generally cleared out within 2 hours after childbirth [6-8]. A noninvasive prenatal test (NIPT) using cfDNA has proven to be highly sensitive and specific for detecting trisomy 21 in both high- and low-risk groups $[1,2,9,10]$. At present, there are about five major companies providing commercial NIPT services globally (Fig. 1). NIPT using cfDNA is now available through these providers in more than 60 countries. According to business reports, over 500,000 NIPT studies on women with a high risk for fetal aneuploidy were performed in the United States in 2013 [11]. These companies have published several articles showing that their own method is very effective for screening for trisomy 21,18 , and 13

\footnotetext{
Received: 15 July 2015, Revised: 13 August 2015, Accepted: 12 October 2015, Published: 31 December 2015

${ }^{*}$ Corresponding author: Kunwoo Kim, M.D.

Hamchoon Women's Clinic, 10 Seochojungang-ro 8 gil, Seocho-gu, Seoul 06643, Korea.

Tel: +82-2-522-0123, Fax: +82-2-522-2388, E-mail: mdkkw@hamchoon.com

Conflict of interest: Hamchoon Women's Clinic is a main distributor of an SNP-based NIPT (Panorama ${ }^{\mathrm{TM}}$ test) in Korea. The author is an employee of this clinic.

(ㄷ) This is an open-access article distributed under the terms of the Creative Commons Attribution Non-Commercial License (http://creativecommons.org/licenses/by-nc/4.0/) which permits unrestricted non-commercial use, distribution, and reproduction in any medium, provided the original work is properly cited.

(c) Copyright 2015 by the Korean Society of Medical Genetics 
$[1,2,10,12-18]$. However, there has been no well-controlled, large-scale study comparing the products of all 5 companies in terms of test accuracy and efficiency. In this article, I review the differences and advantages of single nucleotide polymorphism (SNP)-based NIPTs in comparison to other methods.

\section{NIPT Using cfDNA}

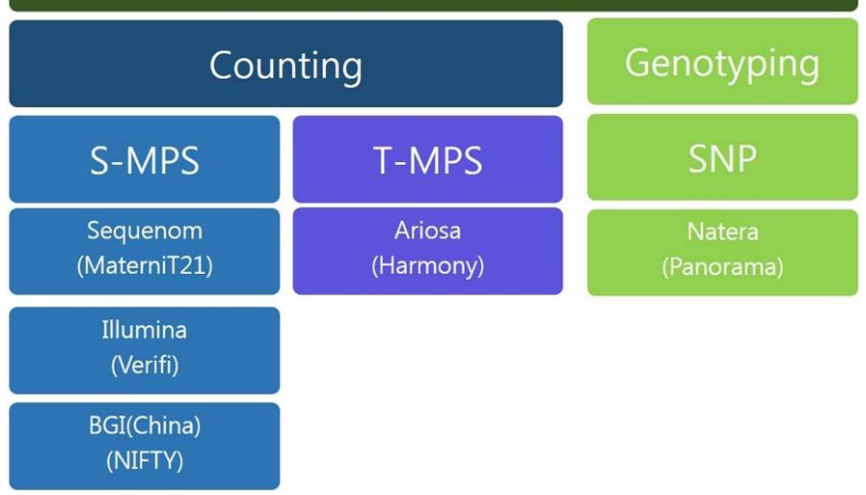

Fig. 1. Classification of different noninvasive prenatal test (NIPT) methods. cfDNA, cell-free DNA; S-MPS, shotgun massively parallel sequencing; T-MPS, targeted massively parallel sequencing; SNP, single nucleotide polymorphism. Sequenom: San Diego, USA; Illumina: San Diego, USA; BGI: Beijing Genomics Institute, Shenzhen, China; Ariosa: San Jose, USA; Natera: San Carlos, USA.

\section{Counting Methods of the NIPT Using cfDNA [11]}

\section{Shotgun massively parallel sequencing}

After extraction and amplification of whole cfDNA from the maternal plasma, shotgun massively parallel sequencing (S-MPS) is conducted based on the sequencing and subsequent counting of large numbers of DNA fragments in the plasma while allocating them to their chromosome of origin. With this method, evidence of aneuploidy is obvious when there is a relative excess (trisomy) or deficit (monosomy) in any particular chromosome of interest compared to the number expected.

\section{Targeted massively parallel sequencing}

Targeted massively parallel sequencing (T-MPS) is similar to S-MPS but includes an extra step that selectively amplifies only the chromosomal regions of interest (for example, chromosomes 21,18 , and 13), and then calculates whether there is an excess for one particular chromosome relative to another. A benefit of the T-MPS methodology is the lower sequencing cost because this method avoids having to sequence all regions.

\section{Principle of the SNP-based NIPT $[19,20]$}

SNPs are normal genetic variations that can be used to

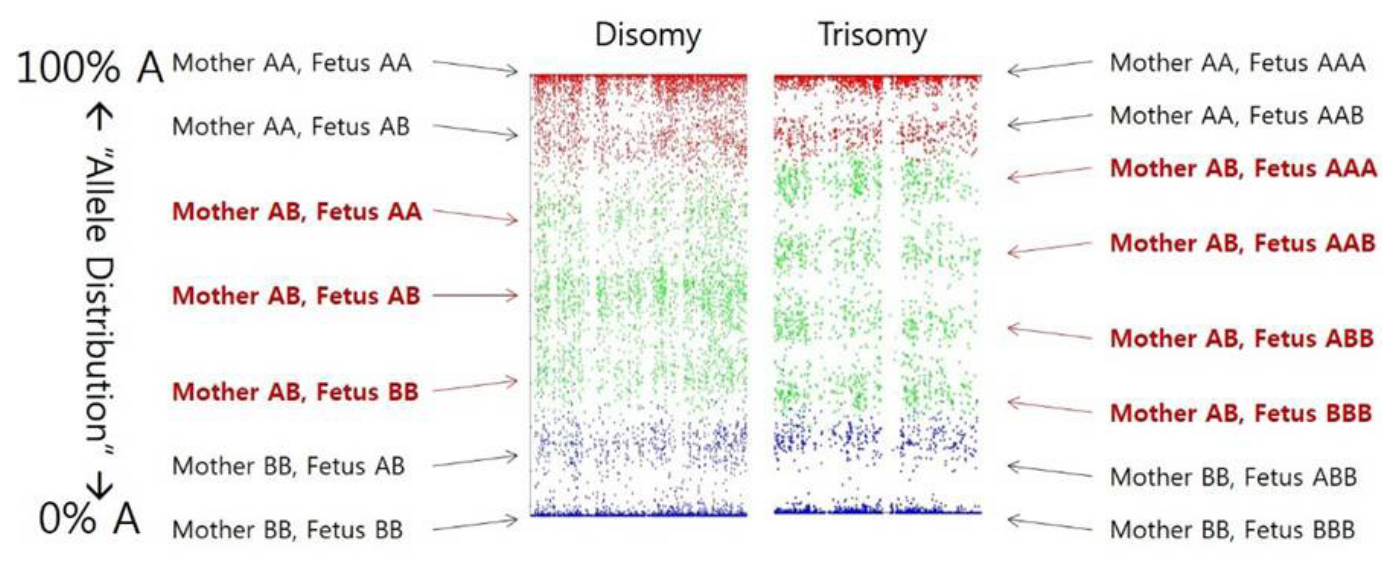

\section{$\leftarrow$ Individual SNP Positions $\rightarrow$}

Fig. 2. Simplified principle of the single nucleotide polymorphism (SNP)-based noninvasive prenatal test. Each dot represents one SNP and the sum of both the maternal and fetal contribution. SNPs are biallelic and thus have only 1 of 2 DNA bases possible (designated as A and B). There is only one SNP per location going from left to right (as though the chromosome is lying on its side), and there is a total of $\sim 3,000$ SNPs per chromosome (this could be visualized if the image were spread out). The top of the profile represents a situation of $100 \% \mathrm{~A}$ and $0 \% \mathrm{~B}$. Continuing down the profile, the frequency of $A$ decreases and that of $B$ increases until reaching the case of $100 \% B$ and $0 \% A$ at the bottom. SNPs in red represent those for which the mother's genotype is $A A$. The top line is the case in which the baby is also $A A$ (thus, the case of $100 \% A$ ). The second line shows the case of $75 \% A$ and $25 \% B$ (the baby genotype is $A B$ ). Green SNPs are those for which the mother's genotype is $A B$. Again, the percentage of $B$ increases from the first to the third line. Blue SNPs are those for which the mother's genotype is BB. Again, the percentage of B increases until the final line, where both the baby and mother are 100\% B. When the mother's SNP is heterozygous (green SNPS), an extra band appears when the fetus is trisomic. However, the band positions are dependent on the allele frequency and fetal fraction. When the mother's SNP is homozygous (red and blue SNPs), one band is shifted when the fetus is trisomic. The extent of this shift is dependent on the allele frequency and fetal fraction. Not all SNP profile results are visually conclusive, which is why an algorithm is needed for interpretation [21]. 
distinguish between any two individuals. Applying SNP analysis to the NIPT helps to determine the difference between parent and child DNA as well as variations in copy number (Fig. 2) [21]. The currently available SNP-based NIPT evaluates 19,488 SNPs and then determines the relative quantitative contributions of maternal and fetal DNA in the maternal plasma. Multiplex polymerase chain reaction amplification of plasma DNA (comprising a mixture of maternal and fetal DNA) and the buffy coat (only maternal DNA) for SNP sequences is followed by direct sequencing to identify which of the amplified products are present. Given that a mother passes on one of each chromosome pair to her children, the chromosomal condition can be classified as monosomy (only one copy from the mother or the father), normal (one copy from each parent), trisomy (an extra copy from either parent), or uniparental disomy (UPD, both copies received from the mother or the father). The Nextgeneration Aneuploidy Test Using SNPs (NATUS) algorithm uses the sequencing data from the mother and father (note that an optional paternal buccal mucosa swab for SNP analysis can improve the reporting rate but does not affect accuracy). This test also incorporates data from the Human Genome Project, which can reveal where crossovers are most likely to occur, since these are region-specific events. Multiple hypotheses for a chromosome of interest are possible: monosomy (a copy from either parent), disomy (one copy from each parent or UPD), or trisomy (with the extra chromosome being either maternal or paternal in origin). Then, millions of sub-hypotheses are established based on all of the potential crossover points. The fetal/maternal DNA pattern is compared to the expectations of each of these sub-hypotheses to find the closest match using a Bayesian-based maximum likelihood statistical method.

\section{Advantages of the SNP-based NIPT}

\section{Less dependency on the fetal fraction}

The fetal fraction represents the proportion of cfDNA in the maternal plasma that is of fetal origin [22], and is one of the most important components of the NIPT. The fetal fraction varies among individuals and factors; for example, it tends to be lower earlier in pregnancy, and can vary with maternal weight $[23,24]$. In the counting methods of the NIPT, the test accuracy is highly dependent on the fetal fraction. If the fetal fraction in maternal plasma is less than $8 \%$, the detection rate of trisomy 21 is about $75 \%[25,26]$. Thus, the main cause of a false-negative result with the counting methods is a low fetal fraction. Although the fetal fraction is the most critical part of an NIPT, similar to cell counting in fluorescence in situ hybridization, most of the counting-based NIPTs, except for the Harmony ${ }^{\text {TM }}$ test (Ariosa Diagnostics, Inc., San Jose, CA, USA), do not report the fetal fraction. By contrast, the SNP-based NIPT is less dependent on the fetal fraction. Indeed, the rate of trisomy 21 detection is not reduced even if the fetal fraction is 4-8\%. Given that approximately 1 out 3 commercial samples have a fetal fraction less than $8 \%$, this distinction is important. The counting methods tend to produce incorrect results from samples with a very low fetal fraction $(<4 \%)$, whereas the SNP-based method tends to make no call in such cases. No call is preferred to a false-negative result, as a no-call result simply requires a redraw and retest, whereas a miscall can result in serious problems and missed diagnoses.

\section{Reduced effect of $\mathrm{GC}$ amplification bias}

GC pairs have 3 bonds between them, whereas A/T pairs only have 2 bonds. When there are fewer or more GC pairs than average, the chromosomes do not amplify in the expected manner during the amplification/sequencing process. The reason is that a variable frequency of the GC pairs can create more or fewer hairpin loops in the DNA. Thus, when the DNA polymerase attempts to bind to the sequence for amplification, it is prevented from binding at the regions with hairpin loops. This is problematic for the counting method, which relies on comparison to reference chromosomes, and therefore any variation in amplification reduces the detection rates. Chromosome 21 contains the optimum median amount of GC pairs. The GC content of chromosome 18 is lower than but similar to that of chromosome 21. Nevertheless, both of these chromosomes tend to amplify close to the expected amount of DNA. By contrast, chromosome 13 has the second-lowest GC content in the genome (after chromosome 4, and with a similar amount as the $X$ chromosome) [27]. Consequently, amplification is variable, thus throwing off the ratios from expectations by amplifying either more or less than the true amount of chromosome 13. This situation decreases the sensitivity of detection for trisomy 13 or monosomy $X$ compared to that for trisomy 21 or trisomy 18 with the counting method $[25,28]$. However, the SNP-based method is not affected by this amplification bias, since it does not require comparison to a reference chromosome; thus, the results are not dependent on the amount of DNA in a chromosome but rather on the specific sequence of the DNA $[13,19]$. 


\section{Effective differentiation between maternal and fetal cfDNA}

The SNP method can readily distinguish between maternal and fetal free-floating DNA by analyzing the buffy coat that contains purely maternal DNA. This allows for establishment of a maternal-only SNP profile. In contrast, the counting method amplifies and sequences both the maternal and fetal DNA, without differentiating between them. This distinction is particularly important for conditions such as monosomy $X$; for example, when it is difficult to determine whether the mother is a mosaic for $\mathrm{XO}$. $\mathrm{X}$ chromosome loss $(\mathrm{XCL})$ is dependent on maternal age; $X C L$ is present in less than $1 \%$ of women below the age of 25 , and the rate of incidence increases with age after this point in a logistic quadratic manner [29]. Therefore, in such cases, the counting method could yield a false-positive result. One study demonstrated that $8.56 \%$ of the positive results for sex chromosome aneuploidies determined with the counting method were revealed to in fact be false positives due to maternal mosaicism [30]. Therefore, without analyzing maternal DNA separately, the counting methods may conclude a fetal sex anomaly. The SNP method analyzes the specific maternal DNA contribution, which helps to decrease the rate of false positives related to maternal mosaicism and copy number variants [31].

\section{Detects a vanishing twin}

Although cfDNA generally goes away within 2 hours after delivery, if a fetus remains in the uterus due to co-twin demise, apoptosis of the placenta occurs, which releases cfDNA from the vanishing twin into the maternal circulation. Even up to 8 weeks post-demise, this vanishing twin DNA can be detected and potentially lead to false-positive results with the counting method. The SNP method can detect the possibility of vanishing twin/triploidy, which would alert the doctor to perform an ultrasound. Studies have indicated that presence of a vanishing twin is one of the major causes of false-positive results given that the demised twin is more likely to be chromosomally abnormal. One study showed that more than 15\% of the false- positive results were associated with the presence of a vanishing twin [15]. The SNP method is the only method that can pick up this additional haplotype, and thus prevent false positives [32].

\section{Detects triploidy}

One major disadvantage of the counting method is that it cannot detect triploidy. Two studies with the counting method confirmed that 8 cases of triploidy were called as 'normal', and thus had a $100 \%$ false negative rate $[15,28]$. As mentioned above, the SNP method does not require comparison to a reference chromosome. Indeed, it was the only test to correctly identify 4/4 cases of paternal triploidy in two blinded studies [33]. Even though most cases of triploidy will miscarry spontaneously, the incidence is $1 / 1,000$ at 10 weeks. In particular, paternal triploidy carries a risk for a partial molar pregnancy. Early diagnosis of triploidy could be helpful for managing these pregnant women [34].

\section{Limitations of the SNP-based NIPT}

The SNP-based method might not be applicable to cases of multiple pregnancy. However, a new algorithm for handling multiple pregnancies is under development. Furthermore, this method is not currently applicable to ovum-donated pregnancy. The NATUS algorithm is too computationally complex to validate other algorithms. Therefore, to add a new target would require intensive work on the SNP method (i.e., addition of new SNPS, primers, interpretations).

\section{Conclusion}

The well-known causes of false positives in an NIPT are a vanishing twin, maternal chromosomal abnormalities (especially sex chromosome mosaicism), and confined placental mosaicism. Of these causes, only the SNP-based NIPT can detect a vanishing twin and maternal abnormalities. Moreover, because the

Table 1. Comparison of test performance with different noninvasive pretatal test methods [11]

\begin{tabular}{lcccccrrr} 
& \multicolumn{2}{c}{ S-MPS } & & \multicolumn{2}{c}{ T-MPS } & & \multicolumn{2}{c}{ SNP } \\
\cline { 2 - 3 } & DR & FPR & DR & FPR & & DR & FPR \\
\hline Trisomy 21 & $99.0(97.7-99.7)$ & $0.19(0.11-0.33)$ & & $99.4(96.4-100)$ & $0.01(0-0.09)$ & $100(96.8-100)$ & $0(0-0.29)$ \\
Trisomy 18 & $97.6(92.8-99.5)$ & $0.15(0.08-0.28)$ & & $97.9(92.2-99.9)$ & $0.08(0.03-0.19)$ & $96.4(80.8-100)$ & $0.09(0-0.54)$ \\
Trisomy 13 & $89.2(74.7-96.3)$ & $0.32(0.20-0.51)$ & & $81.8(51.2-96.0)$ & $0.06(0-0.25)$ & $100(79.7-100)$ & $0(0-0.28)$ \\
All above aneuploidies & $98.2(96.9-99.0)$ & $0.21(0.16-0.29)$ & & $98.2(95.7-99.9)$ & $0.05(0.02-0.10)$ & $99.2(95.1-100)$ & $0.03(0-0.18)$ \\
\hline
\end{tabular}


SNP-based NIPT is less dependent on the fetal fraction, lower false-negative and false-positive rates are expected with the SNP method compared with the counting method (Table 1). However, it is important to note that NIPTs are not direct diagnostic tests but rather advanced screening tests. Although generally designated as fetal cfDNA, the primary source of the cfDNA tested is in fact placental trophoblast cells. Therefore, confined placental mosaicism, which is estimated in 1-2\% of all 10-12 week gestations, impacts all NIPTs [35].

It is clear that when an NIPT is implemented in a clinical setting, the influence of mosaicism cannot be ignored, and its impact on false-positive and false-negative results should be addressed. Therefore, pre-test counseling and post-test counseling by experts is the most important process when performing an NIPT. Because false-positive test results can occur, confirmation with amniocentesis or chorionic villi sampling is recommended. Patients also need to be aware that a negative test result does not ensure an unaffected pregnancy; falsenegative test results can also occur. In the US, approximately $6.2 \%$ of women with high-risk results chose to terminate the pregnancy without invasive test confirmation [10].

I hope that in the future, decisions to terminate a pregnancy will not be based on only NIPT results without a confirmation test. Given a normal NIPT result, maternal serum alphafetoprotein screening or ultrasonographic assessment for open neural tube defects should continue to be offered.

\section{References}

1. Bianchi DW, Parker RL, Wentworth J, Madankumar R, Saffer $C$, Das $A F$, et al; CARE Study Group. DNA sequencing versus standard prenatal aneuploidy screening. N Engl J Med 2014;370:799-808.

2. Norton ME, Jacobsson $B$, Swamy GK, Laurent LC, Ranzini AC, Brar $H_{\text {, }}$ et al. Cell-free DNA analysis for noninvasive examination of trisomy. N Engl J Med 2015;372:1589-97.

3. Lo YM, Corbetta N, Chamberlain PF, Rai V, Sargent IL, Redman CW, et al. Presence of fetal DNA in maternal plasma and serum. Lancet 1997;350:485-7.

4. Flori E, Doray B, Gautier E, Kohler M, Ernault P, Flori J, et al. Circulating cell-free fetal DNA in maternal serum appears to originate from cytoand syncytio-trophoblastic cells. Case report. Hum Reprod 2004;19: 723-4.

5. Faas $B H$, de Ligt J, Janssen I, Eggink $A$, Wijnberger LD, van Vugt JM, et al. Non-invasive prenatal diagnosis of fetal aneuploidies using massively parallel sequencing-by-ligation and evidence that cell-free fetal DNA in the maternal plasma originates from cytotrophoblastic cells. Expert Opin Biol Ther 2012;12 Suppl 1:S19-26.

6. Lo YM, Zhang J, Leung TN, Lau TK, Chang AM, Hjelm NM. Rapid clearance of fetal DNA from maternal plasma. Am J Hum Genet 1999;64:218-24.

7. Nygren AO, Dean J, Jensen $T J$, Kruse $S$, Kwong $W$, van den Boom $D$, et al. Quantification of fetal DNA by use of methylation-based DNA discrimination. Clin Chem 2010;56:1627-35.

8. Sikora A, Zimmermann BG, Rusterholz C, Birri D, Kolla V, Lapaire O, et al. Detection of increased amounts of cell-free fetal DNA with short PCR amplicons. Clin Chem 2010;56:136-8.

9. Gil MM, Quezada MS, Revello R, Akolekar R, Nicolaides KH. Analysis of cell-free DNA in maternal blood in screening for fetal aneuploidies: updated meta-analysis. Ultrasound Obstet Gynecol 2015;45:249-66.

10. Dar P, Curnow KJ, Gross SJ, Hall MP, Stosic M, Demko Z, et al. Clinical experience and follow-up with large scale single-nucleotide polymorphism-based noninvasive prenatal aneuploidy testing. Am J Obstet Gynecol 2014;211:527.e1-527.e17.

11. Benn P. Non-invasive prenatal testing using cell free dna in maternal plasma: recent developments and future prospects. J Clin Med 2014; 3:537-65.

12. McCullough RM, Almasri EA, Guan X, Geis JA, Hicks SC, Mazloom AR, et al. Non-invasive prenatal chromosomal aneuploidy testing--clinical experience: 100,000 clinical samples. PLoS One 2014;9:e109173.

13. Pergament $E_{1}$ Cuckle $H$, Zimmermann $B$, Banjevic M, Sigurjonsson $S$, Ryan $A$, et al. Single-nucleotide polymorphism-based noninvasive prenatal screening in a high-risk and low-risk cohort. Obstet Gynecol 2014;124:210-8.

14. Porreco RP, Garite TJ, Maurel $K$, Marusiak B, Ehrich $M$, van den Boom $D_{1}$ et al. Noninvasive prenatal screening for fetal trisomies $21,18,13$ and the common sex chromosome aneuploidies from maternal blood using massively parallel genomic sequencing of DNA. Am J Obstet Gynecol 2014;211:365.e1-12.

15. Futch T, Spinosa J, Bhatt $S$, de Feo E, Rava RP, Sehnert AJ. Initial clinical laboratory experience in noninvasive prenatal testing for fetal aneuploidy from maternal plasma DNA samples. Prenat Diagn 2013; 33:569-74.

16. Song $Y$, Liu C, Qi H, Zhang Y, Bian $X$, Liu J. Noninvasive prenatal testing of fetal aneuploidies by massively parallel sequencing in a prospective Chinese population. Prenat Diagn 2013;33:700-6.

17. Zhang $H_{1}$ Gao $Y$, Jiang $F, F u$ M, Yuan $Y, G$ uo $Y$, et al. Non-invasive prenatal testing for trisomies 21, 18 and 13: clinical experience from 146,958 pregnancies. Ultrasound Obstet Gynecol 2015;45:530-8.

18. Song K, Musci TJ, Caughey AB. Clinical utility and cost of non-invasive prenatal testing with cfDNA analysis in high-risk women based on a US population. J Matern Fetal Neonatal Med 2013;26:1180-5.

19. Zimmermann B, Hill M, Gemelos G, Demko Z, Banjevic M, Baner J, et 
al. Noninvasive prenatal aneuploidy testing of chromosomes 13, 18, $21, X$ and $Y$, using targeted sequencing of polymorphic loci. Prenat Diagn 2012;32:1233-41.

20. Nicolaides $K H$, Syngelaki A, Gil M, Atanasova V, Markova D. Validation of targeted sequencing of single-nucleotide polymorphisms for noninvasive prenatal detection of aneuploidy of chromosomes 13, 18, 21, $X_{1}$ and Y. Prenat Diagn 2013;33:575-9.

21. Hall MP, Hill $M$, Zimmermann $B$, Sigurjonsson $S$, Westemeyer $M$, Saucier J, et al. Non-invasive prenatal detection of trisomy 13 using a single nucleotide polymorphism- and informatics-based approach. PLoS One 2014;9:e96677.

22. Ashoor G, Syngelaki A, Poon LC, Rezende JC, Nicolaides KH. Fetal fraction in maternal plasma cell-free DNA at 11-13 weeks' gestation: relation to maternal and fetal characteristics. Ultrasound Obstet Gynecol 2013;41:26-32.

23. Nicolaides KH, Syngelaki A, Ashoor G, Birdir C, Touzet G. Noninvasive prenatal testing for fetal trisomies in a routinely screened firsttrimester population. Am J Obstet Gynecol 2012;207:374.e1-6.

24. Chiu RW, Chan KC, Gao Y, Lau VY, Zheng W, Leung TY, et al. Noninvasive prenatal diagnosis of fetal chromosomal aneuploidy by massively parallel genomic sequencing of DNA in maternal plasma. Proc Natl Acad Sci U S A 2008;105:20458-63.

25. Palomaki GE, Kloza EM, Lambert-Messerlian GM, Haddow JE, Neveux $L M$, Ehrich $M$, et al. DNA sequencing of maternal plasma to detect Down syndrome: an international clinical validation study. Genet Med 2011;13:913-20.

26. Canick JA, Palomaki GE, Kloza EM, Lambert-Messerlian GM, Haddow JE. The impact of maternal plasma DNA fetal fraction on next generation sequencing tests for common fetal aneuploidies. Prenat Diagn 2013;33:667-74.

27. Karolchik D, Hinrichs AS, Furey TS, Roskin KM, Sugnet CW, Haussler
D, et al. The UCSC Table Browser data retrieval tool. Nucleic Acids Res 2004;32:D493-6.

28. Bianchi DW, Platt LD, Goldberg JD, Abuhamad AZ, Sehnert AJ, Rava RP; MatErnal BLood IS Source to Accurately diagnose fetal aneuploidy (MELISSA) Study Group. Genome-wide fetal aneuploidy detection by maternal plasma DNA sequencing. Obstet Gynecol 2012; 119:890-901.

29. Russell LM, Strike $P$, Browne $C E$, Jacobs PA. X chromosome loss and ageing. Cytogenet Genome Res 2007;116:181-5.

30. Wang $Y$, Chen $Y$, Tian F, Zhang J, Song Z, Wu Y, et al. Maternal mosaicism is a significant contributor to discordant sex chromosomal aneuploidies associated with noninvasive prenatal testing. Clin Chem 2014;60:251-9.

31. Snyder MW, Simmons LE, Kitzman JO, Coe BP, Henson JM, Daza RM, et al. Copy-number variation and false positive prenatal aneuploidy screening results. N Engl J Med 2015;372:1639-45.

32. Curnow K, Wilkins-Haug L, Ryan A, Kırkızlar E, Stosic M, Hall MP, et al. Detection of triploid, molar, and vanishing twin pregnancies by a single-nucleotide polymorphism-based noninvasive prenatal test. Am J Obstet Gynecol 2015;212:79.e1-9.

33. Nicolaides $\mathrm{KH}$, Syngelaki $\mathrm{A}$, del Mar Gil M, Quezada MS, Zinevich Y. Prenatal detection of fetal triploidy from cell-free DNA testing in maternal blood. Fetal Diagn Ther 2014;35:212-7.

34. Simon AL, Su B, Demko Z, Rabinowitz M, Harmon ER, Gross SJ. Detection of complete molar pregnancy by single-nucleotide polymorphism-based non-invasive prenatal testing. Ultrasound Obstet Gynecol 2015;46:506-7.

35. Grati FR, Malvestiti F, Ferreira JC, Bajaj K, Gaetani E, Agrati $C_{\text {, et al. }}$ Fetoplacental mosaicism: potential implications for false-positive and false-negative noninvasive prenatal screening results. Genet Med 2014;16:620-4. 